\title{
Cancer testicular bilateral: presentación de cuatro casos
}

\author{
Planelles Gómez J, Beltrán Armada JR, Tarín Planes M, Vergés Prosper A, Rubio Tortosa I, \\ Gil Salom M.
}

Servicio de Urología. Hospital Universitario Dr. Peset. Valencia.

Actas Urol Esp. 2007;31(10):1117-1122

\section{RESUMEN}

\section{CANCER TESTICULAR BILATERAL: PRESENTACIÓN DE CUATRO CASOS}

El cáncer testicular bilateral suponen el 2-5\% de todos los tumores de testículo, aunque actualmente esa prevalencia va aumentando. La edad media de aparición es de los 15-35 años y el $75 \%$ de ellos se presenta metacrónicamente.

Suele haber hallazgos histológicos similares en ambos testículos, siendo el tipo histológico más frecuente el seminoma.

El factor de riesgo más importante en el desarrollo del cancer testicular es la presencia de neoplasia intratubular de células germinales.

El tratamiento de elección es la orquiectomía radical, aunque en algunos casos seleccionados se puede realizar una cirugía conservadora del testículo

Presentamos 4 casos atendidos en nuestro servicio, analizamos los factores de riesgo, manejo de los tumores y realizamos una revisión de la literatura médica.

Palabras claves: Cáncer testicular. Bilateral. Neoplasia intratubular de células germinales.

\section{ABSTRACT}

\section{BILATERAL TESTICULAR CANCER: A REPORT OF FOUR CASES}

Bilateral testicular cancer represents from 2 to $5 \%$ of all testicle tumors, even though this prevalence nowadays is increasing. The median age of presentation was from 15 to years and in approximately $75 \%$ of them occurred metachronously.

There used to be concordance in histological findings between both testicles, being seminoma the most common histological type.

The most important risk factor in the development of testicular cancer is the presence of intratubular germ cell neoplasia.

Radical orquiectomy is the treatment to choose, although in some carefully selected patients testis-sparing surgery may be considered.

We present four cases attended in our medical service, we analize the risk factors, management of tumors and a medical literature review is done.

Keywords: Testicular cancer. Bilateral. Intratubular germ cell neoplasia.

$\mathrm{E}^{\prime}$ carcinoma testicular supone, aproximadamente, el $1 \%$ de los tumores malignos en los hombres. El tumor de Células Germinales es el más frecuente y la edad media de aparición es de los 15 a los 34 años siendo el proceso maligno más frecuente en este rango de edad.

La incidencia de los tumores testiculares bilaterales es rara siendo del $2 \%$ al 5\%. Pese a eso, el ries- go de desarrollar un segundo tumor en un paciente con antecedentes de tumor testicular es mayor que el de la población general de contraer un tumor de Células Germinales. El 75\% aproximadamente de estos tumores bilaterales son metacrónicos.

El tipo histológico más común en los tumores testiculares bilaterales es el Seminoma y es frecuente la concordancia histológica. 
La Neoplasia Intratubular de Células Germinales, carcinoma in situ o neoplasia testicular intraepitelial se presenta en el 90\% de los testículos con cáncer y en el 5\% de los testículos contralaterales sanos, por ello se lo considera el principal factor de riesgo para desarrollar un tumor testicular.

Presentamos cuatro casos de tumores testiculares bilaterales, 3 metacrónicos y 1 sincrónico, así como su manejo terapéutico, que fueron tratados en nuestro servicio.

\section{CASO CLÍNICO 1}

Paciente de 25 años que, durante ingreso en el servicio de Medicina Interna por fiebre vespertina de 3 días de evolución, se le descubre incidentalmente mediante estudio ecográfico tumoración en testículo derecho.

Como antecedentes personales, refiere no tener alergias medicamentosas. Herniorrafia inguinal bilateral.

Refiere tumoración indolora de un año de evolución que ha ido aumentando progresivamente.

A la exploración física se palpa aumento del testículo derecho con tumoración indolora en polo superior.

Ante la sospecha de tumor testicular se le realizan diferentes pruebas complementarias. En la Ecografía testicular, se aprecia tumoración en testículo derecho de $78 \mathrm{~mm}$ x $57 \mathrm{~mm}$ x $61 \mathrm{~mm}$ heterogénea, sólida compatible con tumor testicular primario. Teste izquierdo normal. Tomografía axial computerizada (TAC): tumoración testicular derecha con adenopatías retrocavas y paraaórticas $>2$ y $<5$ cm. Lactato deshidrogenasa (LDH): 1890 UI/I; alfafetoproteína (AFP): $51 \mathrm{ng} / \mathrm{ml}$; beta-gonadotropina coriónica (Beta-HCG): $23 \mathrm{mUI} / \mathrm{ml}$.

Se sometió a orquiectomia inguinal derecha con informe de Anatomía Patológica de Carcinoma Embrionario de $7 \mathrm{~cm}$ que sustituye parénquima y epidídimo con implantes en cordón y alcanza albugínea. Diagnosticado de carcinoma embrionario de testículo derecho T3N2MO, estadio II con riesgo intermedio por elevación de $\mathrm{LDH}$, completó el tratamiento con quimioterapia según el esquema BEP (bleom<icina, etopósido y cisplatino) de 4 ciclos.

A los 4 años, y estando libre de enfermedad en las revisiones de control, acude a Urgencias por tumoración dolorosa en testículo izquierdo.

A la exploración física se aprecia, monorquio izquierdo, tumoración indurada a nivel del polo inferior del epididimo izquierdo.

Dentro de las exploraciones complementarias destaca: Lactato deshidrogenasa (LDH): 347 UI/I; alfafetoproteína (AFP): 2,4 ng/ml; beta-gonadotropina coriónica (Beta-HCG): 0,1 mUI/ml. Radiografía de tórax: Sin hallazgos patológicos. Ecografía testicular: Tumoración testicular de 51x19x22 mm de tamaño, con ecoestructura heterogénea y con flujo doppler color, que afecta además a cuerpo y cola de epidídimo. Calcificación en vertiente anteroinferior de testículo. Tomografía Axial Computerizada toraco-abdomino-pélvico: Hígado, bazo y páncreas normales. Riñones de morfología y densidad normales. No adenopatías ni cadenas ganglionares.

Bajo la sospecha de segundo tumor de células germinales se le realiza orquiectomía radical izquierda vía inguinal, siendo le resultado de la Anatomía Patológica de Seminoma clásico de 3,2 cm de diámetro que infiltra parénquima testicular extendiéndose a epidídimo y cordón espermático.

El postoperatorio cursó sin complicaciones.

Se remite al Servicio de Oncología para valoración de tratamiento adyuvante y control. Dado que el diámetro máximo del tumor es de 3,2 cm y no afecta a la rete testis, se opta por el tratamiento conservador de control.

Tres meses después, en Tomografia Axial Computerizada de control, se detecta adenopatía retroperitoneal izquierda aumentada de tamaño (3,5 cm), con mal plano de separación con el psoas que atrapa el tercio medio del uréter causando hidronefrosis grado I. En las analiticas sanguíneas de control: hemograma, bioquímica y hemostasia dentro de los parámetros de la normalidad excepto por Creatinina de 1,2 mg/dl; lactato deshidrogenasa=552 UI/I, alfafetoproteína y beta-gonadotropina coriónica dentro de la normalidad.

Se diagnostica de recidiva retroperitoneal y se somete a tratamiento con quimioterapia (etopósido y cisplatino de 4 ciclos).

Actualmente el paciente lleva 3 años libre de enfermedad en los controles periódicos que se le realizan en los servicios de Urología y Oncología. 


\section{CASO CLÍNICO 2}

Paciente de 29 años, es remitido a nuestro servicio para completar estudio tras acudir al centro de especialidades por tumoración indolora en su testículo izquierdo de un mes de evolución sin otra sintomatología. Se le detecta en la ecografia que se le realiza en el centro de especialidades un testículo izquierdo hipogénico y disminuido de tamaño que dificulta su valoración, y un testículo derecho con parénquima heterogéneo sospechoso de neoplasia.

No refiere alergias medicamentosas y tampoco tiene antecedentes personales de interés.

A la exploración física se palpa un teste izquierdo hipotrófico. El teste derecho se presenta algo aumentado de tamaño. Ambos no dolorosos, de consistencia normal y de superficie regulares. Epidídimos y cordones sin hallazgos patológicos.

Se le solicitan marcadores tumorales que resultan en una alfa-feto proteína de $2.7 \mathrm{ng} / \mathrm{ml}$ con una beta-hCG de $4.2 \mathrm{mUI} / \mathrm{ml}$.

Durante el ingreso se realiza nuevas exploraciones complementarias, y en la ecografia con flujo Doppler se aprecia un teste derecho de $49 \mathrm{~mm}$ x $36 \mathrm{~mm}$ x $26 \mathrm{~mm}$ con lesión hipoecoica, de bordes lobulados, heterogénea, que ocupa prácticamente todo el testículo, y que presenta aumento del flujo Doppler. Y un teste izquierdo de 32 $\mathrm{mm} \times 22 \mathrm{~mm}$ x $20 \mathrm{~mm}$ hetrogéneo con lesión focal ligeramente hipoecoica de aproximadamente $9 \mathrm{~mm}$ con sutil flujo Doppler. Además, microlitiasis testiculares bilaterales.

Al TAC toraco-abdomino-pélvico con contraste, donde no se identificaron adenopatías ni metástasis en ninguno de los territorios visualizados.

Con el diagnóstico de tumor testicular bilateral, se le realizó una orquiectomía bilateral vía inguinal. Al mismo tiempo comenzó tratamiento hormonal sustitutivo.

En la Anatomía patológica informan de testículo izquierdo atrófico con múltiples focos microscópicos de Seminoma clásico asociado a amplias áreas de Neoplasia Intratubular de Células Germinales (NICG) con ausencia de infiltración tumoral en albugínea, rete testis, epidídimo o infiltración vascular. El testículo derecho presenta tumoración con características de
Seminoma clásico que ocupa prácticamente todo el parénquima, asociado a focos de Neoplasia Intratubular de Células Germinales (NICG) y focos de atrofia en el testículo restante. Túnica albuginea, rete testis, epidídimo y borde quirúrgico libres de tumor.

El tratamiento se completó con dos ciclos de quimioterapia con carboplatino.

Actualmente lleva aproximadamente 3 años y medio libre de enfermedad.

\section{CASO CLÍNICO 3}

Paciente de 29 años que es remitido a nuestra consulta tras cuadro de dolor en teste derecho 3 meses atrás que remitió con tratamiento antiinflamatorio. Un mes después tuvo un nuevo cuadro de dolor en testículo derecho que fue diagnosticado de orquiepididimitis pero que no se resolvió con tratamiento médico.

Como antecedentes personales sólo destaca el ser alérgico a las Sulfamidas. No antecedentes urológicos de interés.

A la exploración física se palpa tumoración en polo posteroinferior de teste derecho indolora.

Ante la sospecha de tumor testicular se le solicita una analítica completa sanguínea con marcadores tumorales y una ecografía testicular, siendo los resultados de AFP $36.4 \mathrm{ng} / \mathrm{ml}$ y una Beta-hCG $4.2 \mathrm{mUI} / \mathrm{ml}$. En la ecografia se observa una tumoración de $23 \mathrm{~mm}$ en polo inferior de teste derecho de características no quísticas sospechosa de neoplasia.

Se le interviene quirúrgicamente mediante una orquiectomía radical inguinal derecha cuya Anatomía Patológica informa de Tumor de Células Germinales mixto de $2 \mathrm{~cm}$ formado por Carcinoma Embrionario y focos de Teratoma. No invasión vascular ni linfática. Albugínea, cordón espermático y epidídimo libres de tumor.

Al ser un Tumor Germinal No Seminomatoso en Estadio I, se decide de mutuo acuerdo con el paciente no recibir tratamiento adyuvante con quimioterapia y seguir un control observacional estricto.

$\mathrm{Al}$ paciente se le realizan los controles periódicos según protocolo del nuestro hospital permaneciendo libre de enfermedad en las técnicas de imagen, clínica y analíticamente durante 7 años y medio hasta que en un estudio ultrasonográfico de 
control se le detecta microcalcificaciones en testículo izquierdo único con nódulo hipoecoico de 7 $\mathrm{mm} \times 9 \mathrm{~mm}$, también con calcificaciones y sin aumento de flujo Doppler que es compatible con tumor testicular. Además se observa un varicocele izquierdo.

La exploración física resulta anodina, y los marcadores tumorales están dentro de la normalidad.

Tras orquiectomía inguinal izquierda, la Anatomía Patológica informa de Seminoma de $0,5 \mathrm{~cm}$ que infiltra cápsula sobre testículo atrófico con extensa Neoplasia Intratubular de Células Germinales. No infiltración vascular ni linfática.

Se completó el tratamiento con quimioterapia $\mathrm{y}$ tratamiento hormonal sustitutivo.

Actualmente lleva 4 años libre de enfermedad.

\section{CASO CLÍNICO 4}

Paciente de 25 años que es remitido a nuestra consulta tras descubrírsele en estudio ultrasonográfico masa hipoecoica sugestiva de neoplasia en teste izquierdo.

El paciente consultó en el centro de especialidades por percibir desde hacía unos días un aumento indoloro del testículo izquierdo.

Como antecedentes personales destaca el haber padecido testículo derecho en ascensor en la infancia. A los 9 meses sufrió un cuadro de meningitis que le dejó un déficit neurológico permanente y crisis comiciales residuales. Además, apendicectomía y tenotomía aquílea en la infancia.

Ya en nuestra consulta, se le solicita nueva ecografia, marcadores tumorales y estudio de extensión mediante Tomografía Axial Computerizada. Los resultados de las pruebas fueron de una beta-HCG de $5.7 \mathrm{mUI} / \mathrm{ml}$, con la lactato deshidrogenasa y la alfafetoproteína normales. En la ecografia se observa masa de $8 \mathrm{~cm}$ en teste izquierdo con múltiples nódulos hipoecoicos compatible con tumor testicular, además microcalcificaciones en testículo derecho. No se apreciaron adenopatías o metástasis en el TAC toraco-abdomino-pélvico.

Se le intervino mediante orquiectomía radical izquierda por vía inguinal y el análisis por Anatomía Patológica informó de Seminoma clásico que no infiltra a la albugínea ni al cordón, tampoco existe invasión vascular o linfática.

Con el diagnóstico de Seminoma clásico en Estadio I se opta por un tratamiento conservador. A los 10 meses, en un estudio ultrasonográfico de control, se evidencia un nódulo hipoecoico en testículo derecho sospechoso de neoplasia. La exploración física y los marcadores tumorales son normales.

Se procede a la orquiectomía radical derecha y el estudio por parte de Anatomía Patológica informa de Seminoma de tipo clásico con focos de Neoplasia Intratubular de Células Germinales de aproximadamente $4.5 \mathrm{~cm}$. Tractos vasculares, albugínea y cordón espermático libres de tumor. Se inicia además el tratamiento hormonal sustitutivo.

A los tres meses se detecta un aumento de la beta-HCG de hasta $4.5 \mathrm{mUI} / \mathrm{ml}$, con el resto de marcadores tumorales normales, y se solicita nuevo TAC toraco-abdomino-pélvico que informa de masa retroperitoneal de $3 \mathrm{~cm}$ x $8 \mathrm{~cm}$ paraaórtica por debajo de la arteria renal izquierda que se extiende hasta la bifurcación ilíaca. Así mismo, en situación más anterior, se ve otra imagen de aspecto nodular de $2 \mathrm{~cm} \times 2 \mathrm{~cm}$ con un centro necrótico y captación de contraste periférico.

Con el diagnóstico de recaída adenopática abdominal de seminoma clásico, comienza el tratamiento con 4 ciclos de Etopósido y Cisplatino.

Actualmente lleva aproximadamente 7 años libre de enfermedad.

\section{DISCUSIóN}

La incidencia de los tumores testiculares bilaterales es de entre 2-5\%. Esta cifra ha aumentado en la actualidad debido, principalmente, al desarrollo de las técnicas diagnósticas y al avance en las pautas de quimioterapia que produce un aumento de la supervivencia de los pacientes $^{1}$. La edad media de aparición, al igual que en los unilaterales, de los 15-35 años.

El $75 \%$ de estos tumores aproximadamente aparecen metracrónicamente, siendo la media de tiempo de aparición del segundo tumor de unos cinco años ${ }^{2}$.

Todos los pacientes con tumores testiculares unilaterales tienen un riesgo relativo de unas 35 veces más elevado que el resto de la población de 
desarrollar tumores de células germinales ${ }^{3}$, incluso años después del primer tumor, y aunque hayan recibido tratamiento con quimioterapia, lo cual reduce el riesgo pero no lo erradica del todo ${ }^{4}$.

La concordancia histológica entre tumores es de aproximadamente el $50 \% \%^{4,5}$, y el tipo histológico más frecuente es el seminoma clásico, siendo su prevalencia más alta incluso que en los tumores unilaterales primarios. El tipo histológico no parece influir en la incidencia, aunque existen autores que defienden que la presencia de seminoma aumenta la probabilidad de desarrollar tumores testiculares bilaterales ${ }^{3,6}$.

Se han identificado a lo largo de la literatura diversos factores de riesgo potenciales como la atrofia testicular, infertilidad, criptorquidia, antecedentes familiares de tumor testicular. Este riesgo aumenta si coinciden 2 factores o más.

Con el desarrollo de las nuevas tecnologías, se han intentado identificar nuevos factores de riesgo a nivel genético ${ }^{7}$ o alteraciones en los antígenos de histocompatibilidad. Así la bibliografía habla de un aumento significativo del HLA-B14 ${ }^{8}$, sobre todo.

Pero el principal factor que puede predecir la aparición de un tumor testicular es la neoplasia intratubular de células germinales (IGCN, carcinoma in situ o TIN) $)^{1-9}$.

Fue descrito por primera vez por Shakkebaek en 1972, y se considera precursor de tumores de células germinales seminomatosos y no seminomatosos.

Los resultados de la anatomía patológica hablan de la presencia del IGCN en el 90\% de los testículos con tumores germinales y en el $5 \%$ de los testículos contralaterales de los pacientes con tumores testiculares unilaterales. Estos resultados dan a entender que la presencia de la neoplasia intratubular de células germinales es más frecuente incluso que los tumores bilaterales de células germinales. El riesgo de generar una neoplasia a partir de estas lesiones, se ha estimado en el $50 \%$, sobre todo en los primeros 5 años ${ }^{2,4,10,11}$.

El diagnóstico definitivo viene dado por la histología, bien mediante PAAF, aunque la mayoría de los autores dudan de su eficacia por la cantidad insuficiente de muestra, o mediante biopsia ${ }^{4}$.
Dada su epidemiologia, existe bibliografia que recomiendan las biopsias sistemáticas contralaterales durante la orquiectomía de los pacientes diagnosticados de tumores testiculares unilaterales ${ }^{11}$. El debate surge porque se han detectado complicaciones tras biopsias hasta en un $23 \%$ de los casos $\mathrm{y}^{7}$, actualmente, sólo se recomiendan en pacientes con tumor testicular y variables asociadas de atrofia testicular, criptorquidia y/o menor de 30 años.

Las opciones terapéuticas de la IGCN son la cirugía, la radioterapia o la actitud expectante, no se ha demostrado la eficacia de la quimioterapia sistémica ${ }^{1}$.

La cirugía es curativa, pero requiere el tratamiento hormonal sustitutivo de por vida. El tratamiento de elección es la radioterapia aunque con radiaciones de 20 Gy puede causar infertilidad y posibles alteraciones en las células de Leydig. Actualmente se aplican dosis menores de entre 16 y 18 Gy con menos riesgo ${ }^{12}$.

Todo esto hay que valorarlo antes de dar un tratamiento definitivo ya que no existe evidencia definitiva de que toda neoplasia intratubular de células germinales desarrolle cáncer invasivo aunque sí un aumento de riesgo de tener un tumor contralateral.

Además de la exploración física y los marcadores tumorales, los estudios de imagen como la ultrasonografia escrotal, el ECO-TAC de retroperitoneo y abdomen resultan muy útiles para el diagnóstico de los tumores testiculares bilaterales.

La ultrasonografía escrotal es una técnica de imagen diagnóstica simple, no invasiva y efectiva incluso con aquellos tumores muy pequeños o no palpables. No existen signos patognomónicos de la enfermedad, aunque sí imágenes altamente sugestivas que nos puede sugerir incluso, la naturaleza histológica del tumor ${ }^{5,8}$.

El tratamiento de los tumores testiculares bilaterales al igual que los tumores unilaterales, depende de la histologia y el grado de metástasis. Las bases del tratamiento de los tumores testiculares bilaterales fueron definidas por la Internatinal Germ Cell Consensus Classification y viene definida la actitud terapéutica en función de una estratificación de riesgos. No obstante estas bases pueden ser modificadas en algunos casos según las características individuales de cada paciente ${ }^{10}$. 
La terapia de elección es la orquiectomía bilateral, lo que conlleva un tratamiento hormonal sustitutivo de por vida y a plantearse la criopreservación de semen en caso de desear descendencia $^{13}$. Todo ello sin contar con las alteraciones estéticas que en muchos casos requieren la implantación de prótesis. Por supuesto, en función de su estadio asociado a terapia adyuvante si lo requiere.

Por ello, existe bibliografia que defiende, la resección testicular parcial de aquellos tumores con un tamaño inferior a $2 \mathrm{~cm}$ y en casos muy seleccionados. Sus resultados hablan de unos pacientes libres de enfermedad y con una mejor calidad de vida ${ }^{14,15}$.

\section{REFERENCIAS}

1. Park DS, Prow DM, Amato RJ, Ro JY, Logothetis CJ. Clinical characteristics of metachronous bilateral testicular tumors in the chemotherapeutic era. Yonsei Med J. 1999;40(2):137143.

2. Pamenter B, De Bono JS, Brown IL, Nandini M, Kaye SB, Russell JM, et al. Bilateral testicular cancer: a preventable problem? Experience from a large cancer centre. BJU Int. 2003;92(1):43-46.

3. Osterlind A, Berthelsen JG, Abildgaard N, Hansen SO, Hjalgrim H, Johansen B, et al. Risk of bilateral testicular germ cell cancer in Denmark: 1960-1984. J Natl Cancer Inst 1991;83:1391-1395.

4. Muñoz Vélez D, Mus Malleu A, Rebassa Llull M, Ferrutxe Frau J, Gutiérrez Sanz-Gadea C, Riera Marí V, et al. Bilateral germ tumors of the testis. Report of 5 cases and review of the literature. Actas Urol Esp. 2000;24(8):632 639.

5. Dieckmann KP, Loy V, Büttner P. Prevalence of bilateral testicular germ cell tumours and early detection based on contralateral testicular intra-epithelial neoplasia. $\mathrm{Br} \mathrm{J}$ Urol. 1993:71(3):340-345.

6. Hentrich M, Weber N, Bergsdorf T, Liedl B, Hartenstein R, Gerl A. Management and outcome of bilateral testicular germ cell tumors: Twenty-five year experience in Munich. Acta Oncol. 2005;44(6):529-536.
7. Cruz Guerra NA, Mayayo Dehesa T, Cuesta Roca C, Arias Fúnez F, Sánchez Encinas M, Escudero Barrilero A. Testicular embryonal carcinoma with contralateral synchronous intratubular germ cell neoplasia: analysis of a case. Actas Urol Esp. 2000;24(6):491-495.

8. Kratzik C, Aiginger P, Kuber W, Riccabona M, Kührer I, Joos H, et al. Risk factors for bilateral testicular germ cell tumors. Does heredity play a role?. Cancer. 1991;68(4): 916-921.

9. Coogan CL, Foster RS, Simmons GR, Tognoni PG, Roth BJ, Donohue JP. Bilateral testicular tumors: management and outcome in 21 patients. Cancer. 1998;83:547-552.

10. Che M, Tamboli P, Ro JY, Park DS, Ro JS, Amato RJ, et al. Bilateral testicular germ cell tumors: twenty year experience at M. D. Anderson Cancer Center. Cancer. 2002; 95(6):1228-1233.

11. Von der Maase $H$. Is a contralateral testicular biopsy in patients with unilateral germ cell testicular cancer indicated as a routine procedure?. Acta Oncol. 2005;44(6):523525.

12. Cañís Sánchez D, Conde Santos G, Alonso Gracia N, De León Morales E, Arango Toro O, Gelabert Mas A. Diagnosis and terapeutic management in a germinal bilateral tumors. A report of one case and a literature revisión. Actas Urol Esp. 2003;27(2):147-151.

13. Kliesch S. Diagnosis and primary treatment of testicular tumor. Urologe A. 2004;43(12):1494-1499.

14. Mearini E, Bracarda S, Crinò L, Zucchi A, Porena M. Conservative surgery and quality of life in a young patient with synchronous bilateral embryonal carcinoma. Eur. Urol. 1996;29(1):119-121.

15. Demir A, Onol FF, Eren F, Türkeri L. Testis-sparing surgery in an adult with bilateral synchronous seminomatous tumor. Int J Urol. 2004;11(12):1142-1144.

Correspondencia autor: Dr. J. Planelles Gómez

Servicio de Urología

Hospital Universitario Doctor Peset

Avda. Gaspar Aguilar, 90 - 46017 Valencia

Tel.: 963862500

E-mail autor: jorge_planelles@yahoo.es

Información artículo: Original - Cáncer testicular

Trabajo recibido: enero 2007

Trabajo aceptado: febrero 2007 